polarisirtem Lichte, aus welchen dieser Forscher zu der grossen Bedeutung des Wassertransports bei der Fortpflanzung der Contractionswellen concludirt.

Mögen diese Betrachtungen enden mit der Bemerkung, dass die Geschwindigkeit, mit welcher nach $\mathrm{Helmholz}$ u. A. eine Erregung sich den Nerven entlang fortpflanzt, von derselben Grössenordnung ist, als die der Wellenbewegung des Wassers in elastischen Röhren.

Ist am Ende das eigentliche Wesen der Erregung in einer von der plötzlichen Wasserentziehung veranlassten Wellenbewegung der Nervenflüssigkeit zu suchen, der Wellenbewegung ähnlich, welche eine mit Knallgas gefüllte Seifenblase durch Explosion in der Luft zuwegebringt?

Druckfehler der vorigen Abhandlung, Bd. 52. S. 93. Ż. 8 v. o. steht: grösser, lese: kleiner. S. 106. Z. 15 v. u. steht: 0.00012 , lese: 0.0012 und 0.1 , lese: 0.4 .

\title{
Einige Bemerkungen zu Engelmann's Abhandlung über den Ursprung der Muskelkraft.
}

Von

\section{A. Fick.}

In einer jüngst erschienenen, an interessanten neuen Thatsachen reichen Abhandlung ${ }^{1}$ ) bat Engelmano wahrscheinlich zu machen gesucht, dass die Verwandlung chemischer potentieller Energie in mechanisebe bei der Muskelthätigkeit auf thermodynamischem Wege denkbar sei, unbeschadet der Gültigkeit des zweiten Hauptsatzes der mechanischen Wärmetheorie. Der Verfasser sucht dabei an verschiedenen Stellen die Unstichhaltigkeit der Gründe darzuthun, die ich früher geltend gemacht habe gegen die Möglichkeit, den Muskel als eine thermodynamische Maschine aufzufassen. Da ein Schweigen meinerseits den Anschein erwecken könnte, als ob ich

1) Ueber den Ursprung der Muskelkraft von Th. W. Fngelmann. Leipzig, Verlag von Wilhelm Engelmann 1893. 
Einige Bemerk, zu Engelmann's Abhdlg. üb. d. Urspr. d. Muskelkraft. 607

den Betrachtungen Engelmann's zustimmte, so eriaube ich mir, an dieser Stelle einige Bedenken dagegen zu erheben.

Um die ganze Frage anschaulich hervortreten zu lassen, stellen wir uns einen menschlichen Körper vor, der an einem Tage gearbeitet and während der darauf folgenden Nacht geruht hat. Der Körper ist dann nach 24 Stunden wieder merklich genau in demselben Zustande wie zn Anfang, er hat einen "Kreisprocess" durchlaufen. Die einzigen Veränderungen die stattgefunden haben sind: 1. Es ist dureb Verbrennung einer gewissen Menge von Nahrungsstoffen chemische potentielle Energie vernichtet. 2. Es ist eine gewisse Menge von kinetischer Molekularbewegungsenergie entstanderl, die sich als Wärme in der Umgebung des Körpers verbreitet hat, und es ist 3. eine gewisse Menge von potentieller mechanischer Energie entstanden, wenn wir annehmen, dass die Arbeit des Menschen auf Hub einer Last verwendet worden ist.

Soweit entspricht der gedachte Fall genau dem einer Dampfmaschine, die einen Tag gearbeitet, dann etwa auch in der Nacht geruht hat und am Morgen wieder angeheizt ist. Da ist auch die Maschinerie wieder im selben Zustande (alle Temperaturen wieder dieselben etc.), die Veränderungen sind hier auch 1. Vernichtung" von potentieller chemischer Energie durch Verbrennung von Kohle, 2. Entstehung von kinetischer Energie, die als Wärme in der Umgebung verbreitet ist, und 3. Entstehung von potentieller nechanischer Energie, wenn wir annehmen, dass die Arbeit der Dampfmaschine auch zum Heben einer Last verwendet ist.

Fs ist nun erstens nach dem Grundsatze der Erhaltung der Energie klar, dass in beiden Fällen die vernichtete chemische Energie gleich sein muss der Summe der entstandenen Wärme und mechanischen Energie. Wir wissen ferner, dass bei einem arbeitenden menschlichen Körper die entstaudene mechanische Energie zu der gebildeten Wärme sich unter günstigen Bedingungen verhalten kann $=1: 4$ oder dass, wie man sich auszudrücken pflegt, 1/s der ganzen verbrauchten themischen Energic zu mechanischer Arbeit, verwandt werden kamn. Dabei ist ïbrigens die während der angenommenen Ruhezeit verlorene chemisehe Energie und entwickelte Wärme ausser Rechnung zu lassen.

Sollte nun im arbeitenden menschlichen Körper wie in der Danufmaschine die Verwandlung eines Theiles der aufgewendeten chemischen Energie in mechanische auf thermodynamischem Wege 
zu Stande gekommen sein, d. h. sollte die chemische Energie zunächst Wärme - das ist unge ordnete Molekularbewegung erzeugt haben und von dieser ein Theil durch Vermittelung geeigneter Körper in mechanische Energie verwandelt sein, dann müssten die Bedingungen des zweiten Hauptsatzes der mechanischen Wärmetbeorie erfullt sein. Bekanntlich sagt dieser aus: "Wenn in einem Kreisprocesse bei einer absoluten Temperatur $T_{0}$ ein Wärmequantum $Q_{0}$ in mechanische Energie verwandelt werden soll, so muss nothwendig allermindestens ein Wärmequantum $Q$ uibergehen aus einem Körper von der höheren Temperatur $T_{1}$ auf einen Körper von der niederen Temperatur $T_{2}$, so dass die Gleichung erfüllt ist

$$
\frac{Q_{0}}{T_{0}}=Q\left(\frac{1}{T_{2}}-\frac{1}{T_{1}}\right)
$$

Die absolute Temperatur $T_{2}$ kann im mensehlichen Körper nicht niedriger sein als $273^{\circ}+37^{\circ}=310^{\circ}$. Soll nun $Q_{0}=1 / 4 Q$ sein, so muss unter der günstigsten Annahme $T_{0}=T_{1}$ diese absolute Temperatur $387^{\circ}$ oder $114^{\circ}$ der gewöhnlichen 100 theiligen Scala sein. Mit andern Worten: die Annahme, dass die Verwandlung chemischer Energie in mechanische im menschlichen Muskel auf thermodynamischem Wege erfolgt, zieht die Annahme nach sich, dass die ganze vom Körper währęnd der Arbeit nach aussen abgegebene Wärmemenge in den Muskeln von einem 114 Centigrad warmen auf einen $37^{\circ}$ warmen Körper übergegangen sei.

Engelmann meint nun, die Annahme eines solehen Temperaturgefälles im Muskel babe nichts Widersinniges, das Thermometer zeige uns immer nur die Durchschnittstemperatur desselben, und man könne sich wohl denken, dass einzelne im Muskel zerstreut liegende Punkte, wo gerade die Verbrennung im Gange ist, zeitweise glühend heiss wären, ohne dass dies am Thermometer zu erkennen wäre. Engelmann macht zur Unterstutzung dieser Betrachtung darauf aufmerksam, dass bei einer energischen Zuckung in einem Gramme Muskelsubstanz nach meinen Untersuchungen nur 1/4000 Milligramm Brennmaterial zu verbrennen branche, dass ist der viermillionste Theil der ganzen Muskelmasse. Die gerade brennenden Moleküle müssten also verhältnissmässig weit zerstreut in der Muskelmasse liegen.

Ich gebe die Verträglichkeit einzelner zeitweise hoch erhitzter Stellen in der Muskelmasse mit den Ergebnissen der myothermi- 
Einige Bemerk. zu Engelmann's Abhdig. ül. d. Urspr. d. Muskelkraft. 609

schen Untersuchungen gern zu, glaube aber, dass diese Annahme uns einer thermodynamischen Erklärung der Muskelwirkung keinen Schritt näher bringt, und zwar auf Grund folgender Erwägungen. Die vorhin angeführte, den 2 . Hauptsatz der mechanischen Wärmetheorie darstellende Gleichung gilt nur für den Fall eines $u \mathrm{~m} \mathrm{kehr}$ ba ren thermodynamischen Kreisprocesses, analog dem Carnotschen Cyklus. Fiir nicht umkehrbare Kreisprocesse gilt die Ungleichung $\frac{Q_{0}}{T_{0}}<Q\left(\begin{array}{c}1 \\ T_{1}\end{array}-\frac{1}{T_{2}}\right)$. Wir miissen also annehmen, dass die Muskelarbeit sich aus lauter umkehrbaren Kreisprocessen zusammensetzte mit einem Temperaturgefälle von $114^{\circ}$ auf $37^{\circ}$. Die Annahme eines noch viel grösseren Temperaturgefälles zwischen den minimen Verbrennungsherden und der Ungebung, die an sich den beobachtbaren Thatsachen nicht widerspräche, wïrde ibrigens, wie sich aus der weiteren Betrachtung ergeben wird, auch nicht geniigen.

Das Wesen des umkehrbaren thermodynamischen Kreisprocesses besteht bekanntlich darin: der die Verwandlung der Wärme in mechanische Arbeit vermittelnde Körper, z. B. die Laft in der Heissluftmaschine, erleidet die Veränderung, bei der er positive Arbeit leistet - bei der Luft ist es die Ausdehnung - isothermisch bei hoher Temperatur $\left(T_{1}\right)$ und nimmt dabei die erforderliche Wärme aus einem gleich warmen Körper auf. Hierauf verändert er sich unter fernerer Entlastung im selben Sinne weiter, wobei er weitere positive Arbeit leistet, aber er orleidet diese Veränderung ,adiabatisch" d. h. ohne Wäme aufzunehmen oder abzugeben; die hierbei in Arbeit verwandelte Wärme giebt der Körper also aus seinem eigenen Vorrath her, er kühlt sich also ab (auf die Temperaten $T_{2}$ ), nun wird dem vermittelnden Körper durch A ufwand von A r b e it eine entgegengesetate Veränderung aufgezwungen (er leistet dabei also negative Arbeit wie $\%$. B. die Luft bei der Zusammendrickung). Die dabei durch a ufgewendete Arbeit in Körper entstandene Wärme wird ihm entzogen durch ein mit ihm in Berihrung gesetztes Wämereservoir von der niedrigeren Temperatur $\left(T_{2}\right)$, auf die cr sich durch den zweiten Akt abgekïhlt hatte. Der Körper eleidet also diese Veränderung isotherwisch. Endlich wird dem vermittelnden Körper durch äussere Arbeit „adiahatisch" eine Veränderung (z. B. der Luft Zusammendriekung) aufgezwangen, die ihn in den urspringlichen Zustand 
zurückbringt, insbesondere entsteht dabei im Körper selbst durch die äussere Arbeit die Wärmemenge, die nöthig ist, um ihm auch wieder die ursprüngliche höhere Temperatur $\left(T_{1}\right)$ zu ertheilen. Da während der beiden ersten Akte des Kreisprocesses von dem vermittelnden Körper mehr mechanische Arbeit nach aussen geleistet als wäbrend der letzten Akte erlitten wird, so muss ein Theil der von aussen zugefuhrten Wärme bleibend in Arbeit verwandelt sein. Man sieht nun schon an der üblichen graphischen Darstellungsweise eines solchen Kreisprocesses, dass die dabei in Arbeit verwandelte Wärme ein um so grösserer Bruchtheil von der aus einem Körper hoher Temperatur entnommenen Wärme sein kann, je mehr sich der ganze die Verwandlung vermittelnde Körper beim zweiten Akte des Processes adiabatisch abkühlt, mit anderen Worten, je weiter die beiden Temperaturen auseinander liegren, die der ganze vermittelnde Körper im ersten und im dritten Akte besitzt. Die Temperatur der Wärmequelle, aus der der vermittelnde Körper beim ersten Akte die Wärme schöpft, ist an sich nicht maassgebend. Dies ist nur dann der Fall, wenn man sich - wie man es bei den schematischen Betrachtungen zu thun pflegt - vorstellt, dass die Temperatur des vermittelnden Körpers nur unendlich wenig unter der Temperatur der Wärmequelle liegt. Wenn ich nun En gelmann richtig verstehe, so hält er die ganze doppeltbrechende Substanz des Muskels für den die Verwandlung vermittelnden Körper entsprechend der Luft in der Heissluft- oder dem Dampfe in der Dampfmaschine. Diese ganze Masse, die doch wohl zu mindestens 1/3 der Muskelmasse angeschlagen werden darf, und nicht bloss die minimen zerstreuten Verbrennungsherde, misste also bei dem hypothetischen thermodynamischen Kreisprocesse während eines namhaften Bruchtheiles der Zeit eine sebr hoch uiber $37^{\circ}$ liegende Temperatur besitzen. Unter den vorhin gemachten bestimmten thatsächlichen Voraussetzungen z: B. müsste die ganze doppeltbrechende Substanz des Muskels während eines namhaften Bruchtheiles der Zuckungszeit $114^{\circ}$ warm sein. Das könnte aber doch der thermometrischen Beobachtung nicht entgehen. Die an sich nicht unsinnige Annahme, dass an den zerstreuten Orten der Verbrennung vielleicht zeitweise eine Temperatur von $1000^{\circ}$ herrscht, nützt gar nichts zu der Erklärung um die es sich handelt, denn nicht auf das Temperaturgefälle zwischen dem Verbrennungsherde 
Einige Bemerk. zu Engelmann's Abhdlg. äb. d. Urspr. d. Muskelkraft. 611 und dem Körper, der schliesslich die Wärme aufnimmt, kommt es an, sondern den auf das 'Temperaturgefälle, das in der ganzen Masse des vermittelnden Körpers während des zweiten (des ersten adiabatischen) Aktes stattfindet.

Ich glaube durch diese Betrachtung ist anschaulich und absolut zwingend dargethan, dass die Muskelarbeit unmöglich auf thermodynamische Kreisprocesse zurickgefüht werden kann. Ich glaube, man kann der von mir seit vielen Jahren vertretenen Annahme nicht ausweichen, dass bei der Muskelarbeit die chemischen Anziehungskräfte i $\mathrm{m}$ S i n ne des $\mathrm{M} \mathrm{askelzuges} g$ eordnet un ittelbar mechaniseh zar Wirkung kommen. Lis sei mir gestattet, eine Vorstellung genauer auszumalen, wie es dabei zugehen könnte. Sic weicht nicht wesentlich ab von der Vorstellung, die Pfliiger einmal von dem Vorgange gegeben hat ${ }^{1}$ ). In der Muskelfaser sind kleine, durch Zwischenräume getrennte Scheibchen von krystallähnlichem Gefüge übereinander angeordnet. Wir wollen uns nun an der unteren Seite jedes Scheibchens ein C-Atom, an der oberen Seite ein Sauerstoffatom denken und zwar zunächst in soleher Lage, dass die eigentliche chemische Anzichungskraft zwischen dem 0 -Atom dor einen Scheibe und dem C-Atom der darüberliegenden noch nicht wirken kam. Es mag etwa das O-Atom in der Mitte der oberen Fläche, das C-Atom der unteren Fläche mehr zur Seite liegen. Nun werde durch den Reizanstoss in dem oberen Scheibchen das Molekiul, dem das C-Atom angehört, so verschoben, dass es senkrecht uber das 0 -Atom des nächstfolgenden Scheibchens und dadurch ihm so nahe kommt, dass die gewaltige chemische Anziehungskraft zwischen $\mathrm{C}$ and $\mathrm{O}$ wirksam wird. Da aber das ( - und das O-Atom mit den andern Atomen ihrer Scheibchen chemisch verknüpft sind, und die scheib. chen auch mechanisch ziemlich starre Körper sind, so wird die Anziehungskraft nicht, wie es bei freien Atomen der Fall sein wirde, ein heftiges Oscilliren um den gemeinsamen Schwerpunkt bewirken, mit andern Worten Warme erzeugen, sondern das CAtom wird die obere, das O-Atom die untere Scheibe nachzieben, so dass sie sich unter Auspressung der zwischenliegenden Fliissigkeit einander nähern. Da die Masse der Scheibchen vielleicht mehr als das millionenfache von der Masse der einander anzie- 
henden Atome ist, wird die Geschwindigkeit der Bewegung gegen die bei Wärmeschwingungen vorkommenden Geschwindigkeiten gering, aber doch nicht verschwindend klein sein, da eben die chemische Anziehungskraft eine enorme Intensität besitzt. Wenn wir uns denselben Vorgang zwischen jeden zwei Scheibchen denken, so sehen wir, dass er die ganze Sänle der Scheibchen enger zusammenrücken lassen - die Muskelfaser verkürzen - muss. Diese Verkürzung 'würde nun offenbar in's Unbestimmte bestehen bleiben, wenn nicht ein $z w e i t e r$ Process, zu dem der Reizanstoss selbst auch schou den Anstoss geben muss, dem ersten auf dem Fusse folgte. Er muss darin bestehen, dass die an dem gedachten C- und 0-Atome zunächst hängenden Atome aus dem chemischen Gefüge der beiden Scheibchen sich lösen, und dass so ein Molekül in die Zwischenflüssigkeit frei wird. Gleichzeitig muss man sich denken, dass aus dem Inneren der Scheibchen wieder $\mathrm{C}$ - und $\mathrm{O}$ Atome an die Stellen hinrücken, wo die zuerst betrachteten gelegen haben. Die Scheibchen werden dabei selbstverständlich unter dem Einfluss der elastischen Kräfte der Faser selbst und etwaiger dehnender äusserer Kräfte wieder auseinander gezogen - die Faser wird wieder verlängert. Der Verlust an Substanz, den die Scheibchen bei dem Vorgange erlitten haben, wird ihnen dann aus der umspülenden Flüssigkeit ersetzt und alles ist im ursprünglichen Zustande zu neuer Arbeit bereit. Man wird mir hoffentlich nicht zutrauen, dass ich glaube, vorstehend eine geniigende Erklärung der Muskelzusammenziehung und Wiederausdehnung gegeben zu haben. Ich habe nur andeuten wollen, in welcher Richtung nach meiner Meinung eine Erklärung zu suchen ist. Gefunden kann sie erst werden, wenn wir viel tiefere Einsicht in die Natur der chemischen Anziehungskräfte besitzen.

Ich muss mich nun noch gegen die Beweiskraft eines Versuches wenden, in dem Engelmann, wie mir scheint, den experimentellen Beweis dafiir sieht, dass auf thermodynamischem Wege bei einem Temperaturgefälle von nur $2^{0}$ etwa $20 \%$ von der zugeführten Wärme nutzbar in Arbeit verwandelt werden könnten. Engelmann hat gefunden, dass sich eine gequollene Darmsaite beim Erwärmen zusammenzieht. Unter günstigen Umständen kann diese Zusammenziehung bei erheblicher konstant gehaltener Spannung einen namhaften Bruchtheil der ursprünglichen Saitenlänge betragen und es kann also bei diesem Vorgange eine recht ansehn- 
Einige Bemerk. zu Engelmann's Abhdlg. üb. d. Urspr. d. Muskelkraft. 613

liche Arbeit geleistet werden. Engelmann hat ihr. Wärmeäquivalent in einem solchen Versuche unter ganz plausibelen Annalimen zu $20 \%$ der der Saite zugeführten Wärme berechnet. Da er hierbei das Aequivalent der geleisteten Arbeit nicht zugerechnet hat, wäre wohl richtiger etwas über $16 \%$ zu setzen, wenigstens wenn man die von Engelmann in Rechnung gestellte Zahl 0,8 als specifische Wärme der Saite bei konstanter Länge (nicht bei konstanter Spannung) denkt.

Ich habe in Gemeinschaft mit Dr. Schenk diesen"höhst interessanten Versuch nachgemacht und wir haben die Angaben Engelmann's durchaus bestätigt. Engelmann ist aber entschieden im Irthume, wenn er an die soeben erwähnte Berechnung die Sätze anknupft: „Dieser Werth iibertriftt die Maximalleistung "der meisten thermodynamischen Maschinen ganz erheblich. Ich nhalte es nicht fir undenkbar, dass nach dem Principe unseres ., Modelles bei geeigneter Wahl und Zubereitumg des Materiales und -zweckmässiger Anordnung der Lrwärmungs- und Abkiihlungs. bedingungen sich vielleicht kraftmaschinen werden herstelen "Lassen, welche merklich sparsamer als alle bekannten thermody. .namischen Maschinen und vielleicht nicht sehr viel weniger spar„san als ein guter Muskel arbeiten." Dies ist absolut unnöglich. Man mag sich drehen und wenden wic man will, man mag Dampf, Luft oder Darmsaiten als vernittelnden Körper anwenden bei einem Temperaturgefälle von $2^{\circ}$, sagen wir von 77 auf $75^{\circ}$ (in diesem Temperaturintervalle zog sich die Darmsaite in einem Versuche Engelmann's besonders energisch zusammen), wird man nach der allgemein gültigen Formel $\frac{Q_{0}}{T_{0}} \leqq Q\left(\begin{array}{c}1 \\ T_{2}\end{array}-\frac{1}{T_{1}}\right)$ nie mehr als 0,0057 das ist etwa 6 per Mille von der zugetühten Wärme nutzbar, in Arbeit verwandeln können.

Zu dem angefühten Trugschlusse ist $\mathrm{E} n \mathrm{ng} / \mathrm{man}$ n gekommen, weil er nicht beachtet hat, dass eine $t$ e $\mathrm{ch}$ is ch nutzbare Verwandlung von Warme in Arbeit nur durch einen $\mathrm{K}$ reisprocess crzielt werden kann, bei dem der vermittehde Körper sich zu Ende des Processes in demselben Zustande befindet wie zu Anfang, so dass eben der Process beliebig oft wiederholt werden kann. Durch einen einmaligen, dem En grelman n'schen Versuche ähnlichen Akt kann man ja bekanntlich durch Vermittelung eines Gases z. B. nicht bloss $20 \%$, sondern die zanze zugeführte Wärme 
ohne alles Temperaturgefälle in Arbeit verwandeln. Man braucht nur das Gas mit einem unerschöpflichen Wärmereservoir von gleicher Temperatur zu verbinden und ihm durch allmähliche Minderung des Druckes Ausdehnung zu gestatten. Dann ist, wenn wir das Anfangsvolum $v_{0}$, das Endvolum $v_{1}$ und den variabelen Druck $p$ nennen, die geleistete Arbeit $\int_{v_{0}}^{v_{1}} p d v$ und es wird nur eine dieser Arbeit'genau a equivalente Wärmemenge aus dem Reservoir in die Gasmasse ïbergehen.

Man kann iabrigens mit der gequollenen Darmsaite wie mit einer Gasmasse dem Carnot'schen Cyklus genau analoge Kreisprocesse ausfiihren, die theoretisch eine ausnutzbare Verwandlung von Wärme in Arbeit gestatten, bei denen dann freilich der zweite Hauptsat\% der mechanischen Würmetheorie in Kraft bleibt, so dass an eine technische Ausniitzung nicht zu denken wäre, da eine gequollene Darmsaite kein grosses Temperaturgefälle erträgt. In der That ist ja eine gequollene Darmsaite nach Engelmann's Versuchen ein Körper, dessen Länge eine Funktion von Temperatur und Spannung ist, ähnlich wie beim Gase das Volum eine Funktion von Temperatur und Druck ist. Bei der Darmsaite nimmt die Länge bei konstanter Spannung mit wachsender Temperatur ab. Hieraus folgt nach den Grundsätzen der mechanischen Wärmetheorie a priori, dass die Saite, wenn sie sich durch Entlastung zusammenzieht, sicb abkühlt, und dass sie sich durch Dehnung crwärmt. Bei dem sich ganz ähnlich verhaltenden Kautschuk sind, beiläufig yesagt, diese Folgesätze auch experimentell erwiesen. Man kann hiernach einen dem Carnot'schen Cyklus analogen Process von der Saite in folgender Weise durchlaufen lassen. Man giebt zu Anfang der Saite eine bedeutende Spannung und Länge $\left(l_{0}\right)$, umgiebt sie mit einem relativ unerschöpflichen Wärmereservoir von gleicher Temperatur $\left(t_{1}\right)$, man entlastet sie nun bis zu einem gewissen Spannungswerthe, wobei sie sich zusammenzieht. Die dabei verschwindende Wärme wird ihr aus dem umgebenden Reservoir ersetzt, so dass ihre Temperatur konstant bleibt (isothermische Verkürzung). Nun umgiebt man die Saite mit einer für Wärme undurchdringlichen Hülle und gestattet ihr durch weitere Entlastung bis auf eine noch kleinere Länge $\left(l_{1}\right)$ sich zu verkürzen (adiabatische Verkürzung). Hierbei sinkt ihre Temperatur auf 
Einige Bemerk. zu Engelmann's Abhdlg. üb. d. Urspr. d. Muskelkraft. 615 einen Werth, den wir mit $t_{2}$ bezeichnen wollen. In diesen beiden Akten hat die Saite eine Arbeit nach aussen geleistet $=\int_{l_{0}}^{l} s . d l$, wenn wir nit $l$ ihre variabele Länge und mit $s$ ihre variabele Spannung bezeichnen. Diese Arbeit wïrde unter geeigneten Umständen eine Grösse von derselben Ordnung sein, wie die Arbeit in Engelm an n's Versuch. Wir denken uns nun die Saite in Verbindung gesetzt mit einem Wärmereservoir von der Temperatur $t_{2}$ und dehnen sic durch Anfwendung äusserer Arbeit bis zu einer gewissen Länge aus, die sich zum Voraus bestimmen liesse, wenn man die Funktion $l=f(s, t)$ kennte und die einer bernach sich ergebenden Bedingung entspricht (isothermische Dehnung). Hat die Saite diese Lảnge erreicht, so wird das Wärmereservoir fortgenommen und die nichtleitende Hïlle angelegt und nun wird die Saite durch weitere Steigerung der Spannung, also durch Anfwand ausserer Arbeit gedehnt (adiabatische Dehnung). Die soeben besprochene Länge muss so gewählt sein, dass, wenn bei der Dehmung die Länge $l_{0}$ wieder erreicht ist, die Saite auch gerade wieder dic Anfangstemperatur $t_{0}$ erreicht, indem sie sich ja dureh die adiabatische Deh: nung erwärmt. Bei den zwoi letzten Akten wird an der Saite aussere Arbeit geleistet in betrage von $\int_{l_{1}}^{l_{b}} s d$. Da aber die mit den gleichen Längendifferentialen multiplicirten Werthe von $s$ in diesem Integrale alle kleiner sein niissen, als in dem ersten, so sieht man, dass nach Beendigung des Kreisprocesses von der aus dem ersten Reservoir an die Saite abgegebene Wärme ein gewisser Theil bleibend in Arbeit verwaudelt ist. D i eser Process kaun nun, wenn man von den allmählichen bleibenden Veränderungen der Saite absieht, beliebig oft wiederholt werden. Es brancht aber wobl kaum durch eine Zeichnung anschaulich gemacht zu werden, dass der wirklich nutzbar in Arbeit zu verwandelnde Betrag von Wärme sich als ein winziger Bruchtheil der Wärme herausstellen würde, die aus dem ersten in das zweite Reservoir ubergegangen ist, wenn die Temperaturen dieser beiden Reservoire innerhalb der für eine gequollene Saite zulässigen 'Temperaturgrenzen liegen sollen. 